\title{
Design with Multiple Virtualized in Cloud Computing Laboratory
}

\author{
Zhaohui Du \\ Guangdong Vocational Collage Post and Telecom, Guangzhou 510630, China \\ 18998461908@189.cn
}

Keywords: Cloud computing, laboratory, virtual desktop, thin-client.

\begin{abstract}
Cloud computing laboratory is necessary in computer and communication courses teaching. Currently, in many universities and colleges cloud computing laboratory has single function. There are basically two functions: one is the use of using many kinds of software on generic cloud desktop, and the other is the use of training cloud computing skill. Our design improved the utilization and integration of laboratory functions. It combined two functions. The user can select or switch the template to choose the function as he needs. It increased the effectiveness of the laboratory.
\end{abstract}

\section{Introduction}

AS the cloud era approaching, the computer and communication technology developed so fast .There is a challenge before us, which is how to construct the cloud computing laboratory in universities and colleges. The advantage of cloud computing is the integration of resources and improving the utilization of equipment. This advantage cannot be provided by traditional laboratory. More and more universities and colleges will increase the cloud computer major or related courses. Then the cloud computing laboratory construction is very necessary. There is a problem as we built our laboratory. If the laboratory is only used in cloud computing, that leads to lack of computer resources. If the laboratory used in cloud computing and using other software, when we do cloud computing experiments that would damage the system. This thesis is to solve this problem. The design of laboratories contains fifty-five computers.

\section{Laboratory requirement analysis}

The laboratory is equipped with a set of virtualization and cloud computing platform and 56 terminals. It has two functions, one is to provide the basic experiment of all kinds of computer course, and the other is to provide the experiment of deploying a set of virtualized cloud computing system. As the same time, the laboratory should has the following advantages.

Safety and Easy Management. At present, most multimedia laboratory is still used in traditional PC model to manage computer, to restore desktop applications by reductive card. Updating system and deploying the desktop environment need expensive cost. The computers always were attacked by virus. On the other hand, since students operate system at will, that made us update the device frequently. Hardware cost increase. Teachers cannot control of the students' behavior effectively. Many problems exists in the system and management of current experimental teaching. That directly affect the teaching result. Therefore, we must find a unified management method.

Software Diversification. The original laboratory has single function. If we want to change the environment, that will be very complex and takes a long time. It makes the arrangement of curriculum very difficult. The new computer lab requires the ability to achieve most of the experimental curriculum needs, such as Linux, Windows systems management, website development, e-commerce, computer software, website development classes, CAD,PS graphics processing, financial management, office automated management courses. As same time, it can achieve virtualization experiment without affect other software's use. In this laboratory, we should provide a built-in variety of experimental environment, teaching environment, the training environment, and can be 
customized to different environments depending on the course requirements. It should achieve allocate resources according to user needs.

Less Investment. It should achieve allocate resources according to user needs. It should be save the money as we equip and upgrade the device. In cloud systems, the user needs only a thin-client to use high-performance directly. This saves the host costs. If the terminal is fault, we just need to replace it with a new thin-client.

\section{Solution deployment}

The design pattern of cloud computing laboratory is IaaS (infrastructure as a service). Using server virtualization technology, the physical server is divided into a plurality of logical servers. We can manage resource using the server efficiently. Using desktop virtualization, we install the Windows for desktop platform. With this, users can connect to their assigned desktop through any terminal equipment. Scheme is shown in Figure 1.

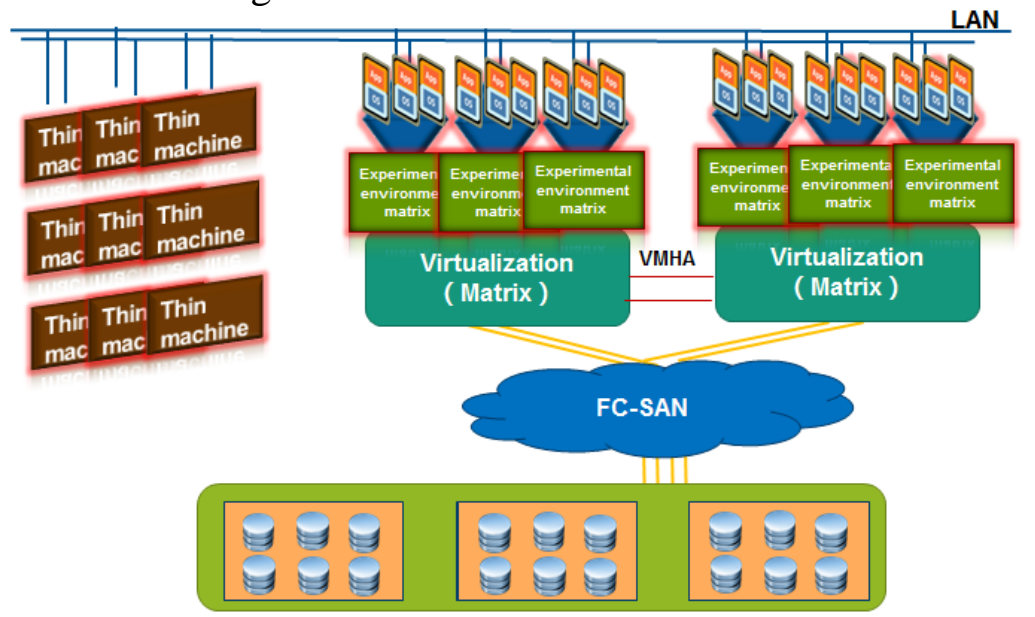

Fig. 1 Laboratory system architecture

\section{Resource requirement}

Using In Virtual Cloud Desktop. Using in virtual Cloud Desktop is primarily use software tools. In this environment should be able to achieve most of the experiment accord computer course's needs.

Using Case Description. A virtual desktop needs to be done, include databases, application development, office applications, graphics software and other applications. A virtual desktop needs such as shown in table 1.All we need are fifty six terminals.

Table 1 Virtual Desktop Resource Analysis

\begin{tabular}{|c|c|c|c|c|c|}
\hline Use Scene & Desktop Type & $\begin{array}{l}\text { Desktop } \\
\text { Persistent }\end{array}$ & $\begin{array}{l}\text { Allocation } \\
\text { Method }\end{array}$ & $\begin{array}{c}\text { Default } \\
\text { Protocol }\end{array}$ & $\begin{array}{c}\text { User data } \\
\text { Persistence }\end{array}$ \\
\hline \multirow{5}{*}{$\begin{array}{l}\text { Computer } \\
\text { Laboratory }\end{array}$} & Automatic & Floating & Link Clone & PCoIP & $\begin{array}{c}\text { Folder } \\
\text { Redirection }\end{array}$ \\
\hline & RAM & CPU & IOPS & $\begin{array}{l}\text { Network } \\
\text { Bandwidth }\end{array}$ & Client Cache \\
\hline & 4GB & $4 \mathrm{VCPU}$ & 40 & $8 \mathrm{Mb} / \mathrm{s}$ & $2 \mathrm{~GB}$ \\
\hline & $\begin{array}{c}\text { Operating } \\
\text { system ,Disk }\end{array}$ & User Data & Suspend File & Page File & Log File \\
\hline & $30 \mathrm{~GB}$ & $15 \mathrm{~GB}$ & 1GB & $3 G B$ & 100MB \\
\hline
\end{tabular}

Resource Requirement Analysis. When we calculate the number of desktop, we accord to concurrent peak using rate of $100 \%$. There is 56 sets in a laboratory. All kinds of resource specific needs is shown in table 2. 
Table 2 Resource Demand of Virtual Desktop Environment

\begin{tabular}{|c|c|c|c|c|c|}
\hline Resol & rce Category & Stand-alone & Number of terminals & \multicolumn{2}{|c|}{ Total } \\
\hline & VCPU & $4 \mathrm{VCPU}$ & 56 & \multicolumn{2}{|c|}{$224 \mathrm{VCPU}$} \\
\hline & RAM & 4GB & 56 & \multicolumn{2}{|c|}{ 224GB } \\
\hline \multirow{5}{*}{ Storage } & user system & 30GB & 56 & \multicolumn{2}{|l|}{$1680 \mathrm{~GB}$} \\
\hline & User Data & 15GB & 56 & \multicolumn{2}{|l|}{$840 \mathrm{~GB}$} \\
\hline & Page file & 3GB & 56 & $168 \mathrm{~GB}$ & \multirow[t]{3}{*}{$2750 \mathrm{~GB}$} \\
\hline & Suspend File & $1 \mathrm{~GB}$ & 56 & $56 \mathrm{~GB}$ & \\
\hline & Log File & $0.1 \mathrm{~GB}$ & 56 & $5.6 \mathrm{~GB}$ & \\
\hline
\end{tabular}

Use of Cloud Computing and Virtual Experimental Environment. Using case description. In this environment is mainly providing students with server virtualization experiments. Four people in each group, we deploy of high-performance server underlying, 28 virtual servers. There are 28 virtual VESXI provide to 14 groups for 56 students. Each group has two virtual servers to complete a series of teaching experiments.

Computing of resource requirements. In this kind of environment, each group achieves high availability experiments with two VESXI. We accord to concurrent peak using rate of $100 \%$. The resource specific needs is shown in table 3.

The total resource calculation. We should put these two parts of resources together to determine the number of resources, at the same time consider the redundancy, it is shown in Table 4.

Table 3 Resource Demand of Cloud Computing Environment

\begin{tabular}{|c|c|c|c|c|c|c|c|c|}
\hline \multirow{2}{*}{ Resources } & \multicolumn{6}{|c|}{ Demand/Group } & \multirow{2}{*}{ Groups } & \multirow{2}{*}{ Total } \\
\hline & $\mathrm{AD}$ & CS & DB & Venter & Composer & Template and Desktop & & \\
\hline VCPU & 4 & 4 & 8 & 8 & 4 & 8 & 14 & 504 \\
\hline Memory & 6 & 6 & 8 & 8 & 6 & 8 & 14 & 588 GB \\
\hline Disk & 50 & 50 & 100 & 50 & 50 & 80 & 14 & 5320 GB \\
\hline
\end{tabular}

Table 4 Calculation of All Resource

\begin{tabular}{ccccc}
\hline Resources & Ordinary Experiment & Virtual Experiment & Redundancy & Total \\
\hline VCPU & 224 & 670 & 96 & 990 \\
Memory & $224 \mathrm{~GB}$ & $870 \mathrm{~GB}$ & $96 \mathrm{~GB}$ & $1190 \mathrm{~GB}$ \\
Disk & $2750 \mathrm{~GB}$ & $7400 \mathrm{~GB}$ & $50 \mathrm{~GB}$ & $10200 \mathrm{~GB}$ \\
\hline
\end{tabular}

The overall allocation of resources. According to the number of resources needs, we should further integration, computer out of the final number required server configuration, concrete results are as following:

CPU Calculation. The number of VCPU is 990, we calculate it according to 1:8. One kernel is equivalent to 8VCPU. Each server has 4 roads. Every road has 8 kernel. So each server has 32 kernels. The final results is we need 4 servers to provide 990 VCPU.

Memory Calculation. The total memory is 1190GB for four servers. Each server configurator with 298GB that is 19 chips with 16GB per chip.

Hard Disk Calculation. The total disk space is 10200GB. We should think about two kinds of components. One kind of is 600G SSD disk, the other is 400G SAS disk. All the disks include 2 SSD disks and 22 SAS disks. Disk must support dual controller, each controller with 16GB cache. Storage support multilevel caching mechanism, to achieve the high frequency of reading data.

\section{System management design}


The experimental environment needs to install the virtual operating system and related software. In this design, we choose the sphere as virtualization products that is the entire desktop terminal architecture. The logic graphs of the system management structure is shown in figure 2.

In underlying system the software we used is sphere that is in a suite of software named "view" . We deploy the virtual machines in the bottom of the server, create a desktop in a virtual machine. So we could have a different desktop used in the class at terminal. By deployed virtualization, 56 virtual desktops are working online at the same time. That achieved switching smoothly, easy to use and management. If we would build a virtual environment, we used virtual server to virtualization again, that is the quadratic virtualization. In this way, we can realize everyone can learn to deploy desktop. The students access cloud desk not only on low-performance machines in the class, but also on notebook, smart phones, thin terminal through the LAN and the WAN. It also provides a platform for students to learn and practice in spare time.

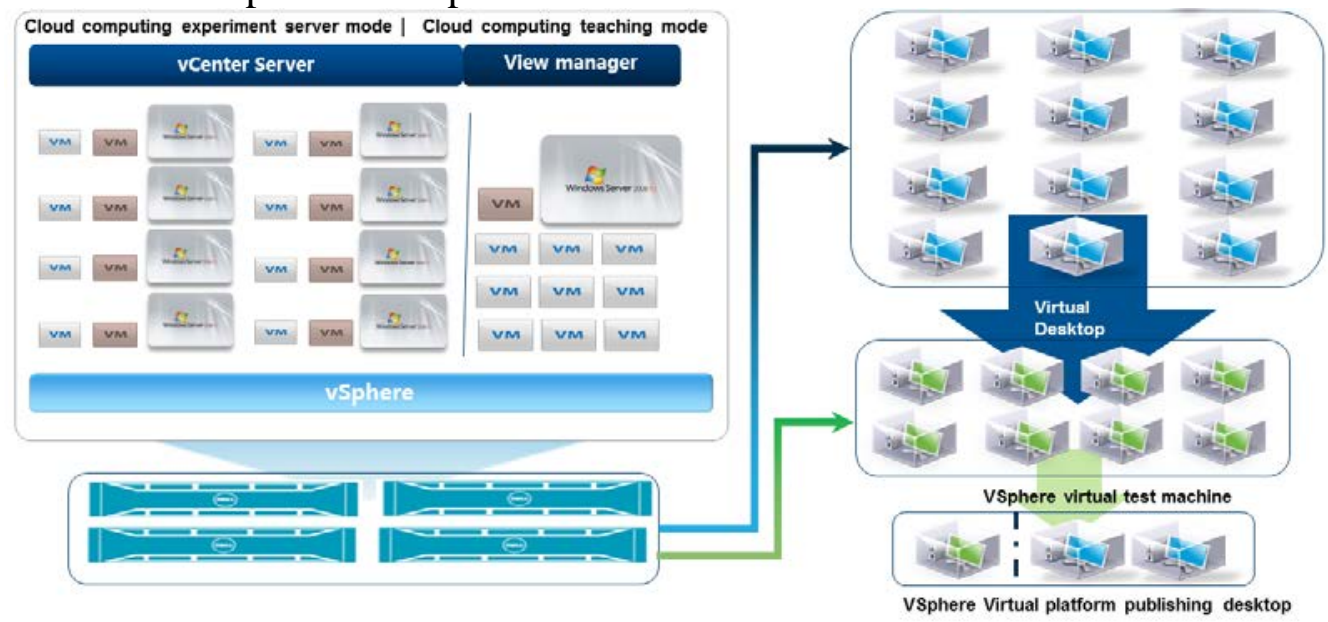

Fig. 2 System logical management structure

\section{Conclusion}

Using of Cloud Computing Laboratory, it can provide convenience for the computer professional teaching practice of real curriculum, at the same time it also enable students to make the cloud platform by themselves. The use of cloud technology laboratory construction, not only can solve the problem of lack of funding infrastructure, but also can make the management organized. Laboratory construction scheme for cloud computing may be further applied. The construction of cloud computing laboratory needs to be promoted. In the university and vocational college, cloud computing will become the important direction and the focus of building information university campus.

\section{References}

[1] Shivaji P., Mirashe , Dr. N. V. Kalyankar , Cloud computing, Journal of computing, 2010, 2(3):78-82.

[2] Feng Dengguo, Zhang Min, Zhang Yan, Xu Zhen. Study on Cloud Computing Security [J]. Journal of Software, 22, 1. P. 71- 83, (2011).

[3] Sun C. Research of E-Commerce Based on Cloud Computing Advances in Computer Science and Information Engineering, p. 15-20, (2012).

[4] Cusumano M. Cloud computing and SaaS as new computing platforms. Communications of the ACM,(2010).53(4):p. 9-27

[5] Kong Yi.Uses of Cloud Computing in Laboratory Construction and Management of University and Colleges[J].China Education Technoloay \&Equipment.Vol.12(2013),p.28-31 
[6] Alshuwaier, F.A., Alshwaier, A.A. and Areshey, A.M. (2012) 'Effective use of cloud computing services in education', Journal of Next Generation Information Technology (JNIT), Vol. 3,No. 4, p.62-77. 